\title{
Tumor-induced myeloid deviation: when myeloid-derived suppressor cells meet tumor-associated macrophages
}

\author{
Stefano Ugel, ${ }^{1}$ Francesco De Sanctis, ${ }^{1}$ Susanna Mandruzzato, ${ }^{2,3}$ and Vincenzo Bronte ${ }^{1}$ \\ IImmunology Section, Department of Pathology and Diagnostics, University of Verona, Verona, Italy. ${ }^{2}$ Section of Oncology and Immunology, Department of Surgery, Oncology and Gastroenterology, \\ University of Padova, Padova, Italy. ${ }^{3}$ Veneto Institute of Oncology IOV-IRCCS, Padova, Italy.
}

\begin{abstract}
The generation of an inflammatory environment is favorable and often decisive for the growth of both primary tumors and metastases. Tumor cells either express membrane molecules or release tumor-derived soluble factors able to alter myelopoiesis. Tumor-reprogrammed myeloid cells not only create a tolerogenic environment by blocking $\mathrm{T}$ cell functions and proliferation, but also directly drive tumor growth by promoting cancer stemness, angiogenesis, stroma deposition, epithelialto-mesenchymal transition, and metastasis formation. In this Review, we discuss the interplay between immunosuppressive and protumoral myeloid cells and detail their immune-regulatory mechanisms, the molecular pathways involved in their differentiation, as well as their potential role as prognostic and diagnostic biomarkers and prospective targets for innovative approaches to treat tumor-bearing hosts.
\end{abstract}

Tumor progression depends on the gradual accumulation of genetic and epigenetic aberrations in cancer cells that also modify the cellular composition of the tumor environment, establishing a state of chronic inflammation characterized by the stromal infiltration of immune cells. Myeloid cells play a critical role in sustaining cancer progression (1). Moreover, inflammatory myeloid cells help to create and fuel the mutagenic pressure underlying the genetic instability of neoplastic cells by both direct mechanisms, such as the production of free-radical compounds (2), and indirect processes, such as the disruption of host defense barriers (3).

Tumor growth is assisted by tumor-associated macrophages (TAMs), the major leukocyte population infiltrating cancers (4). Although macrophages have the potential to attack and eliminate tumor cells, TAMs exhibit many protumoral features that are partly shared by macrophages involved in tissue repair, and they interfere with the function and proliferation of immune effectors (5). Thus, a high frequency of TAMs is associated with poor prognosis in many but not all human tumors (6).

Myeloid-derived suppressor cells (MDSCs) have received increased attention, and their presence and frequency in the blood of patients with tumors is emerging as a potential and simple prognostic marker to monitor clinical outcome and response to therapy (7). MDSCs are characterized by their myeloid origin, heterogeneous cell composition, and ability to negatively regulate adaptive and innate immune responses to cancer. Although TAMs and MDSCs are regarded as separate entities (Figure 1), the boundaries between them are not clearly demarcated, and they share many characteristics (8). TAM accumulation in cancerous tissues is sustained by circulating inflammatory monocytes $\left(\mathrm{CCR} 2^{+} \mathrm{Ly}^{6} \mathrm{C}^{+}\right.$cells

Conflict of interest: The authors have declared the no conflict of interest exists. Reference information: / Clin Invest. 2015;125(9):3365-3376. doi:10.1172/JCI80006. in mice and $\mathrm{CCR} 2^{+} \mathrm{CD} 14^{+} \mathrm{CD} 16^{-}$cells in humans; ref. 9), which are distinct from vessel-patrolling monocytes (Ly6C ${ }^{\text {lo }} \mathrm{CX} 3 \mathrm{CR} 1^{\mathrm{hi}}$ in mice and $\mathrm{CD} 14^{\mathrm{dim}} \mathrm{CD} 16^{-} \mathrm{CX} 3 \mathrm{CR} 1^{\mathrm{hi}}$ in humans). Interestingly, immunosuppressive MDSCs with monocytic features are able to traffic from BM to tumors, mainly through the same chemokine pathway (10). Therefore, the CCR2/CCL2 axis is required for MDSC and TAM accrual and functional specialization. Here, we review the distinctive and common characteristics of TAMs and MDSCs, their role in maintaining cancer growth, and the ongoing development of selective therapeutic approaches.

\section{MDSCs and TAMs result from}

\section{altered myelopoiesis}

The most pervasive and efficient strategy of immune escape likely relies on cancer's ability to create a widespread tolerogenic environment by altering normal hematopoiesis and promoting the expansion of myeloid cells through the constant and progressive release of tumor-derived factors (TDFs), which include metabolites, cytokines, and chemokines (ref. 11 and Figure 2). This "reactive myelopoiesis," leading to MDSC and TAM accumulation, presents marked and distinct molecular features compared with emergency granulopoiesis (12), as emphasized below.

Macrophage composition in different tissues or inflammatory environments depends on a dynamic equilibrium between recruited and tissue-resident macrophages. Tissue-resident macrophages originate at the prenatal stage from the yolk sac and fetal liver (13-15) and acquire selective, tissue-dependent features through the activation of distinctive transcriptional profiles (16-20). During inflammation and under steady-state conditions in some tissues, macrophages are derived from circulating $\mathrm{Ly}_{6 \mathrm{C}}{ }^{+} \mathrm{CCR} 2^{+}$monocytes, as in the case of colonic mucosal macrophages (21).

In cancer, the evidence to date indicates that TAMs are dynamically replaced by circulating precursors. Both the tissue- 
A

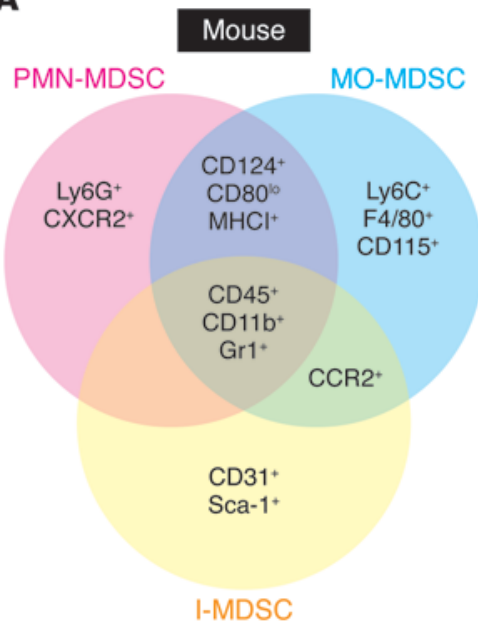

B

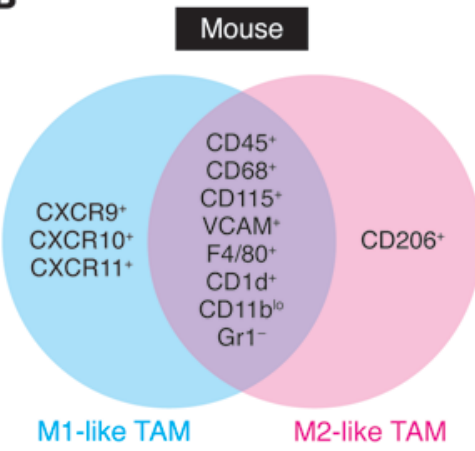

Human

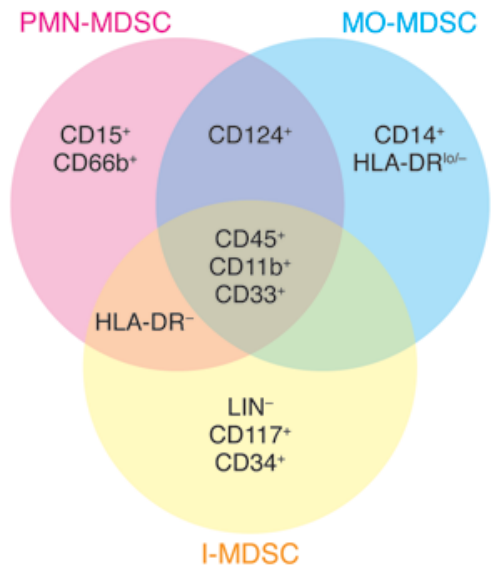

Human

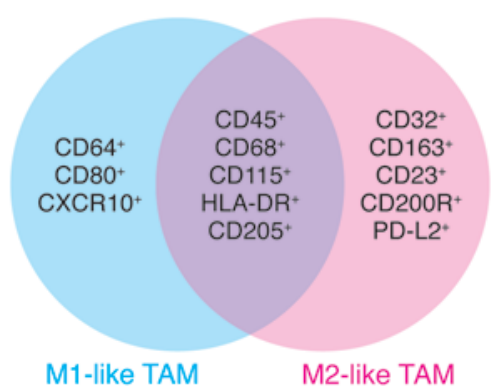

Figure 1. Common phenotypic markers of

MDSCs and TAMs. Several phenotypic markers of mouse and human MDSCs (A) and TAMs (B) have been identified (+ indicates expression, while indicates lack of expression) and used to define specific cell subgroups, such as PMN-MDSCs, MO-MDSCs, and immature MDSCs (I-MDSCs), as well as M1-like and M2-like TAMs, by both cytofluorimetric and immunohistochemical analyses. resident macrophages present in normal mammary tissues and TAMs that develop during tumor progression in the MMTVPyMT breast cancer model are derived from blood-circulating $\mathrm{CCR}^{+}$monocytes, but only TAMs display self-renewal capability (22). In fact, TAM differentiation relies on the NOTCH/recombination signal-binding protein for the $\mathrm{Ig} \kappa \mathrm{J}$ region (RBPJ) signaling pathway and is cell restricted, as genetic ablation of $R b p J$ caused a reduction in both TAMs and tumor growth (22). In the MMTVneu mouse model of autochthonous mammary carcinogenesis, in situ cell division of fully differentiated $\mathrm{CD} 11 \mathrm{~b}^{\mathrm{lo}} \mathrm{F} 4 / 80^{\text {hi }}$ macrophages was the main contributor to the rapid TAM expansion; however, circulating monocyte influx was required in the long

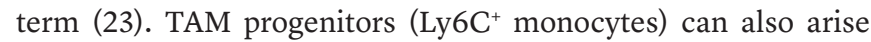
from tumor-induced extramedullary hematopoiesis within the spleen (24), although the relative contribution of BM and spleen to the monocyte reservoir and tumor trafficking is not clear and might be tumor dependent (25).

MDSCs in tumor-bearing hosts: cellular heterogeneity. Normal $\mathrm{CD}_{11} \mathrm{~b}^{+} \mathrm{Gr}^{+}$cells in BM are multipotent cells that can differentiate, depending on the kind and/or extent of cytokine/chemokine stimulation, into cells able to either enhance (e.g., myeloid DCs) or restrain (MDSCs) the immune response $(26,27)$. However, even in tumor-bearing hosts, $\mathrm{BM} \mathrm{CD} 11 \mathrm{~b}^{+} \mathrm{Gr}^{+}$cells are poorly suppressive, while the same cells isolated from liver, spleen, blood, and tumors are fully competent to inhibit T cell activation $(28,29)$. These findings suggest that the BM niche is not permissive for a complete, functional maturation of MDSCs.
As further detailed by Marvel and Gabrilovich (30), mouse MDSCs have been divided into two main subgroups with different phenotypic and biological properties: the monocytic (MO-MDSC) and polymorphonuclear/granulocytic (PMN-MDSC) subsets (31). In tumor-bearing mice, MO-MDSCs ( $\left.\mathrm{Gr} 1^{\mathrm{lo} / \mathrm{int}} \mathrm{CD} 11 \mathrm{~b}^{+} \mathrm{Ly} 6 \mathrm{C}^{\mathrm{hi}} \mathrm{Ly}_{6 \mathrm{G}}{ }^{-}\right)$ are highly immunosuppressive and exert their effects largely in an antigen-nonspecific manner, whereas PMN-MDSCs (Gr1 ${ }^{\text {hi }} C D 11 b^{+}$ Ly $6 \mathrm{C}^{\mathrm{lo}} \mathrm{Ly} 6 \mathrm{G}^{+}$) are moderately immunosuppressive and promote $\mathrm{T}$ cell tolerance via antigen-specific mechanisms $(32,33)$. TDFs induce tumor-infiltrating MO-MDSC differentiation into immunosuppressive TAMs. This conversion is primarily mediated by CSF1 (34), but also by molecular pathways controlled by the hypoxia-inducible factor $1 \alpha$ (HIF-1 $\alpha$ ) (35). HIF-1 $\alpha$ may also be stabilized by the lactic acid that is produced by aerobic glycolysis (Warburg effect) in cancer cells (36). Alternatively, lactic acid can be actively produced in immune-regulatory myeloid cells by cytokine-activated, anerobic glycolysis $(28,37)$.

TAMs in tumor-bearing hosts: cellular plasticity. After arriving at the tumor site, $\mathrm{Ly}_{6 \mathrm{C}^{+} \mathrm{CD} 11 \mathrm{c}^{-} \mathrm{MHCII}} \mathrm{CD} 11 \mathrm{~b}^{\text {hiVCAM }}{ }^{-}$monocytes undergo sequential phenotypical changes characterized by the downregulation of Ly6C and CD11b and the upregulation of MHC class II (MHCII) molecules, VCAM, and CD11c (22). However, TAM differentiation and distribution is not a defined and preserved track but depends on both anatomical location and the tumor stage: cancers with different histology are infiltrated by TAMs with phenotypic and functionally distinct features (38). It is essential to avoid simplified conclusions regarding TAM onto- 


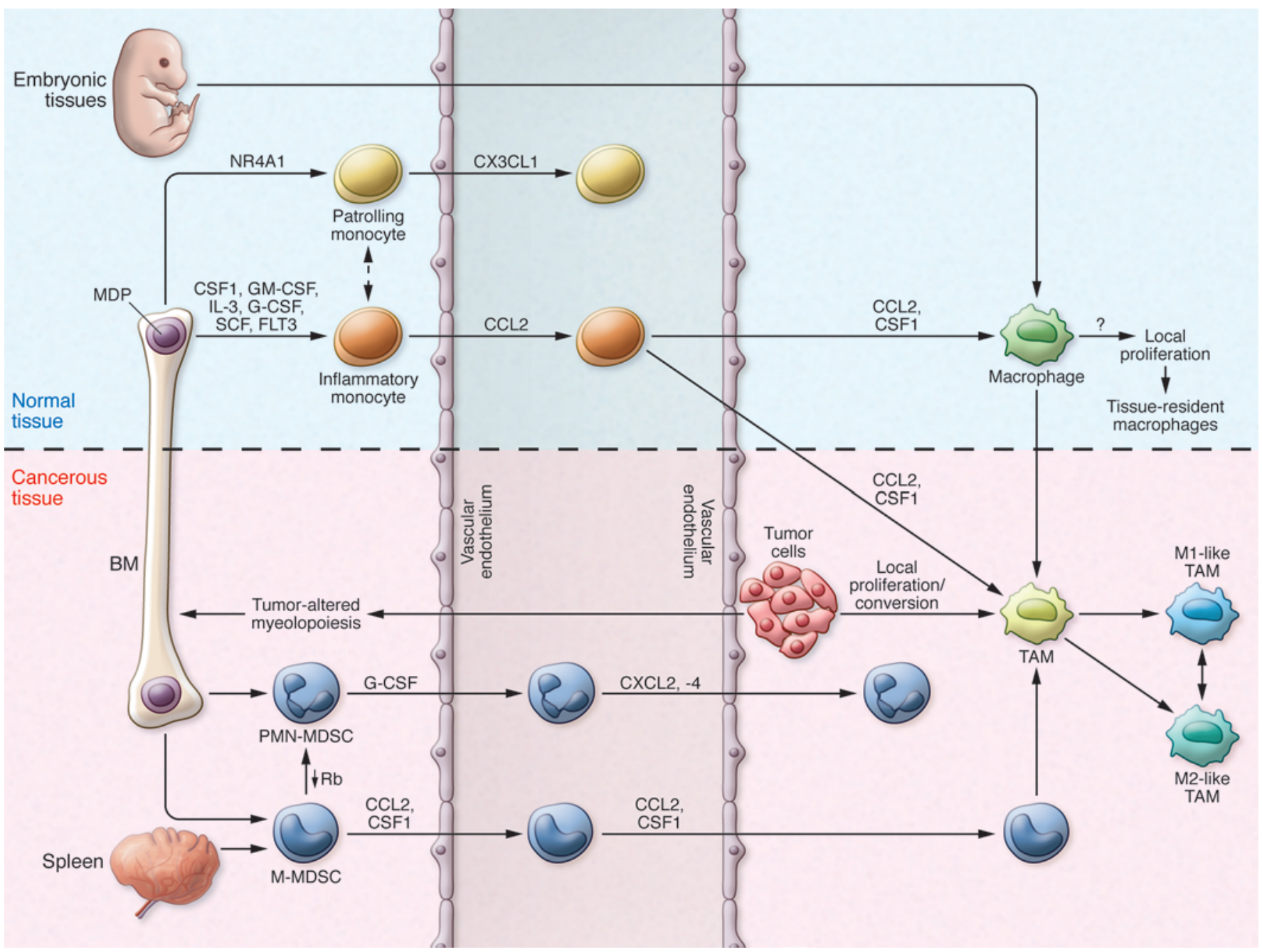

Figure 2. MDSC and TAM development in tumor-bearing mice. Under steady-state conditions, resident macrophages may originate from either embryonic tissues or inflammatory monocytes. Resident macrophages are programmed by local factors, and molecular switches support their differentiation. Circulating monocytes can be divided into two subsets: patrolling monocytes (Ly6C ${ }^{\mathrm{lo}} \mathrm{CX} 3 \mathrm{CR} \mathrm{1}^{\mathrm{hi}}$ ) and inflammatory monocytes (Ly6C $\mathrm{C}^{\mathrm{h}} \mathrm{CD} 11 \mathrm{~b}^{+} \mathrm{CD} 11 \mathrm{c}^{-} \mathrm{MHCII}$ VCAM1-CCR2+), originating from macrophage and DC precursors (MDPs) in BM. Inflammatory monocytes migrate from blood to tissue under the guidance of CCL2/CCR2 chemokine signaling. Tumor cells secrete several factors that modify physiological myelopoiesis, promoting MDP differentiation into

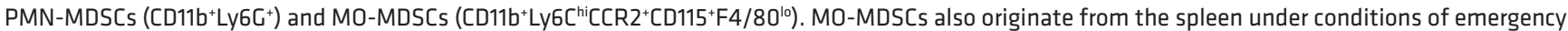
and reactive myelopoiesis. MO-MDSCs and inflammatory monocytes migrate to tumor tissues via CCL2/CCR2 and CSF1 signaling and differentiate into

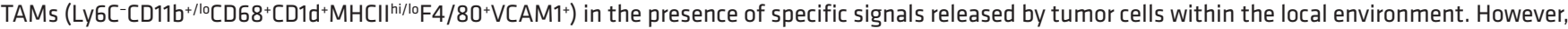
the TAM phenotypic profile depends on cancer histology and stage, which might influence marker distribution. TAMs also proliferate locally, with different rates in various tumors. Furthermore, TAMs are inherently plastic, with an activation state falling along a continuum between the two extremes of M1and M2-like phenotypes. Rb, retinoblastoma.

genetic analysis; for instance, the adoptive transfer of fully differentiated macrophages to alternate tissues demonstrated that the local environment is sufficient to reprogram both the macrophage chromatin landscape and gene expression, similar to what happens to less mature, BM-derived myeloid precursors (20).

The definition of TAM function that is based on a rigid dichotomy in which inducible NOS-positive (iNOS, also known as NOS2) macrophages (M1) are antitumoral and ARG1-positive macrophages (M2) are protumoral is no longer satisfactory and was recently revised (39). The M1 and M2 designations should only describe macrophages activated by either IFN- $\gamma$ and LPS or IL-4 and IL-13, respectively, and M1 and M2 should be viewed as the extremes of a continuum that emphasize the extremes of macro- phage plasticity. M1 and M2 extremes exhibit specific, characteristic expression of metabolic enzymes (iNOS vs. ARG1), cytokines

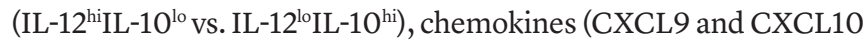
vs. CCL17 and CCL22), and marker genes (Nos2, IL12b, and Ciita vs. Arg1, Retnla, and Chi3l3), as well as transcription factors (NF- $\kappa \mathrm{B}$, STAT1, and IRF5 vs. STAT6, MYC, IRF4, KLF4, and PPAR $\gamma$ ) (39). M1 macrophages are functionally proinflammatory and cytotoxic, whereas M2 macrophages act preferentially in antiinflammatory responses and tissue repair; however, when applied to TAMs, this classification is excessively simplistic and can generate misunderstandings and serious errors in data interpretation. For instance, mammary carcinoma-derived TAMs exhibit M2-related gene expression that is IL-4 independent and primarily orchestrated by 
NOTCH signaling (22) or lactic acid-stabilized HIF-1 $\alpha$ (36). M1-like TAMs are detectable in early-stage cancers as well as in regressing cancers and necrotic areas of growing tumors (40). Furthermore, monocytes isolated from the blood of patients with renal cell carcinoma (RCC) simultaneously express both tumor-suppressing genes, such as TNF and $I L 1 A$, and tumor-promoting genes, such as VEGFA, MMP9, and HIF1A, a mixed profile that was confirmed in macrophages of RCC specimens (41). Thus, TAM classification will require the integration of a multiparameter analysis of cell surface markers, exclusion of ambiguous identifications, and comparison of the TAM transcriptome with the gene profile of resident macrophages isolated from the same tissues (39).

\section{Factors driving TAM and MDSC recruitment, expansion, and activation during tumor growth}

In tumor-bearing hosts, MDSC and TAM generation requires the integration of at least two types of signals: factors that expand myeloid precursors, followed by factors that activate immune-regulatory programs. Myeloid cells are activated and localize to specific tumor areas with different kinetics during primary tumor formation. CSF1, granulocyte-CSF (G-CSF), and granulocyte-macrophage CSF (GM-CSF) are the three chief regulators of myeloid lineage proliferation and differentiation. G-CSF promotes the differentiation of myeloid precursors into PMN-MDSCs. Expansion of Ly6G $\mathrm{G}^{+} \mathrm{PMN}-\mathrm{MDSC}$ occurs very early during tumorigenesis in the MMTV-PyMT mouse model, and these cells are detectable in the blood, spleen, and lungs of mice at the onset of oncogenedriven malignant conversion (42). In this model, tumor-released G-CSF stimulated reactive granulopoiesis at the expense of erythropoiesis by expanding hematopoietic stem cells and granulocyte/macrophage progenitors, but not common myeloid progenitors. This peculiar precursor signature in the $\mathrm{BM}$ is reproduced by either G-CSF or GM-CSF inoculation $(31,42)$ as well as by transplantable, GM-CSF-secreting tumors (31), suggesting a shared action of both cytokines on myeloid progenitors. G-CSF also mediates the lung infiltration of PMN-MDSCs, a step required for the formation of the premetastatic niche (43).

GM-CSF and IL-6 activate the immune-suppressive program in $\mathrm{BM}$-derived progenitors by regulating the $\mathrm{C} / \mathrm{EBP} \beta$ transcription factor (28) and affect myeloid function during very early stages of pancreatic ductal adenocarcinoma (PDAC) progression. After initiation of the transforming program controlled by the active KRAS oncogene in mouse PDAC models, there are progressive waves of myelomonocytic cell recruitment, with $\mathrm{CD} 11 \mathrm{~b}^{+} \mathrm{Gr} 1^{+}$cells and TAMs being among the first to be accrued (44). Along with transformed epithelial cells, $\mathrm{CD}_{11 \mathrm{~b}} \mathrm{Gr1}^{+}$cells contribute to the local release of IL-6 and IL-11, which activate protumoral STAT3 in cancer cells $(45,46)$. Moreover, KRAS-dependent release of GM-CSF primed $\mathrm{CD}_{11} \mathrm{~b}^{+} \mathrm{Gr}^{+}$cells to suppress tumor-specific $\mathrm{CD}^{+} \mathrm{T}$ cells and promoted progression to invasive PDAC; only the blockade of either $\mathrm{GM}-\mathrm{CSF}$ production or $\mathrm{CD} 11 \mathrm{~b}^{+} \mathrm{Gr}^{+}$cell activity restored antitumor immunity (47). Other unknown factors might promote systemic $\mathrm{CD}_{11} \mathrm{~b}^{+} \mathrm{Gr}^{+}$cell expansion in tumors driven by the viral SV40 oncogene, but GM-CSF was nonetheless required for the full in vivo

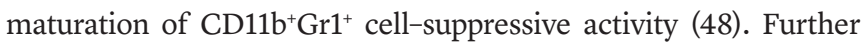
highlighting the role of GM-CSF, mesenchymal breast cancer cells activate TAMs by the combined activity of GM-CSF and lactate; in turn, TAMs release CCL18, which supports epithelial-to-mesenchymal transition (EMT) and metastasis formation (49).

The master factor for TAM recruitment and programming in the tumor microenvironment is CSF1. Genetic deletion of CSF1 either slowed tumor initiation or decreased disease progression and distal metastatic spread, both of which were associated with TAM loss or reduction $(50,51)$. Indeed, elevated CSF1 levels correlated with marked macrophage infiltration in human metastatic breast cancer (52). In addition to CSF1 and CCL2, several other TDFs attract circulating monocytes to the tumor site. For instance, chemokines, such as CCL5, CXCL12, and CX3CL1 (53) as well as growth factors and noncanonical chemotactic peptides, such as VEGF, TGF- $\beta$, bFGF, and the antimicrobial peptide $\beta$-defensin 3 , are involved in monocyte recruitment and macrophage differentiation (54).

IL-4 and IL-13 participate in both TAM and MDSC survival and the acquisition of an immune-suppressive phenotype. They bind different receptors sharing the IL-4R $\alpha$ chain that is responsible for recruiting and phosphorylating STAT6, which induces the transcription of genes involved in the immune-suppressive program, including Arg1 (55). GM-CSF released by mouse and human gliomas upregulate IL-4R $\alpha$ in MDSCs (56), which further fuel a positive loop for MDSC-mediated immune-suppressive activity by releasing IL-13 and IFN- $\gamma$, with the last cytokine maintaining IL-4R $\alpha$ surface expression (57). Accordingly, IL-4R genetic depletion impaired MDSC-dependent immune suppression in vivo (57), and administration of aptamers targeting IL-4R $\alpha$ triggered MDSC and TAM apoptosis and delayed tumor progression (58). Additionally, IL-4 in the tumor microenvironment (secreted by tumor cells or Th2-polarized infiltration T cells) $(59,60)$ induces local macrophages to produce WNT7 $\beta$, thereby promoting tumor invasion (61).

Metabolic environmental signals can also modulate the intratumoral distribution of myeloid cells. Macrophages can survive in a hypoxic environment, but the high lactate levels produced via the Warburg effect can influence their spatial dissemination within specific areas of tumors as well as their dismissal (62). Hypoxia induces semaphorin 3A (SEMA3A), which interacts with a holoreceptor composed of neuropilin 1 (NRP1) and plexin A1/A4 to trigger VEGFR1 phosphorylation and macrophage recruitment (63). A TAM retention signal within hypoxic areas is delivered by SEMA3A through plexin A1/A4; conversely, NRP1 is downregulated in cancer, and its genetic inactivation in macrophages enhances TAM trapping within normoxic areas, resulting in the ablation of their immunosuppressive and proangiogenic activity (63). Partial correction of tumor hypoxia did not affect the relative distribution of TAM subsets or overall M2 marker expression, but rather downregulated the hypoxia-sensitive genes and proangiogenic activity of TAMs residing in the hypoxic areas (64).

\section{Myeloid cells and cancer promotion}

MDSC and TAM activity is not simply a buildup of an immunesuppressive environment that keeps $\mathrm{T}$ cells at bay and protects tumors from the effector arm of the immune system, but includes mechanisms that sustain and promote tumor growth and metastasis (Figure 3), as detailed below.

MDSC- and TAM-induced immune dysfunction. TAMs and MDSCs exert their immunosuppressive effects in an antigen- 


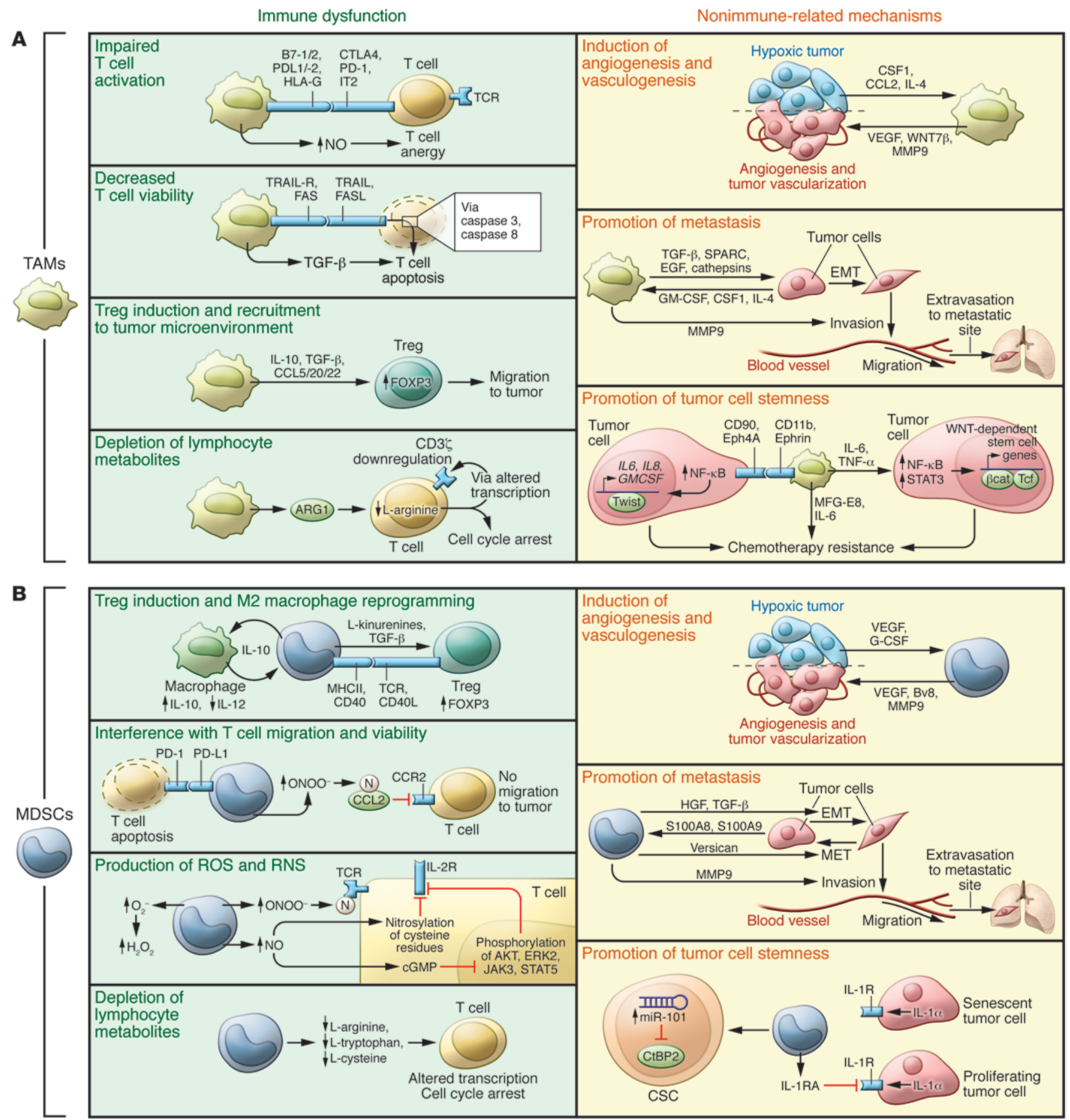

Figure 3. TAM- and MDSC-dependent mechanisms driving tumor progression. TAMs and MDSCs sustain tumor growth, progression, and dissemination by promoting immune dysfunction (green slices) but also by nonimmune-related mechanisms (yellow slices). (A) TAMs alter immune responses in tumor-bearing hosts by four main mechanisms: 1) inhibition of T cell activation; 2) inhibition of T cell viability; 3 ) promotion of Treg induction and recruitment; and 4) consumption of metabolites essential for T cell fitness. TAMs promote tumor angiogenesis and vasculogenesis by the release of VEGF and WNT7 $\beta$, which favor the generation of new blood vessels and sustain metastasis. Finally, TAMs maintain the cancer cell reservoir by secreting IL- 6 and TNF- $\alpha$ and produce MFG-E8 to protect CSCs from chemotherapy. (B) MDSCs inhibit the immune response in tumor-bearing mice by four processes: 1) MDSCs drive the differentiation of immune cells toward regulatory cells; 2) MDSCs interfere with T cell migration and viability; 3) MDSCs alter T cell fitness by turning on intracellular ARG1, NOS2, and NOX2 expression to produce NO, ROS, and RNS (ONOO- $\left.\mathrm{O}_{2}^{-}, \mathrm{H}_{2} \mathrm{O}_{2}\right)$; and 4) MDSCs deplete essential metabolites for T lymphocyte fitness. MDSCs can also promote tumor angiogenesis and vasculogenesis via VEGF and MMP9 secretion. MDSCs produce elevated levels of TCF- $\beta$ and HCF in primary tumors, inducing EMT, and secrete versican in the metastatic niche, promoting MET. Finally, MDSCs maintain tumor cell stemness by both IL-1RA production and by inducing the upregulation of miR-101 in cancer stem cells. cGMP, cyclic GMP; $\beta$ cat, $\beta$-catenin; N, nitrosylated/nitrated; Tcf, HNF1 homeobox A. 
specific and -nonspecific manner, deploying strategies that can be either direct or indirect, with the latter involving the generation or expansion of other regulatory cell populations, such as $\mathrm{CD} 4{ }^{+} \mathrm{CD} 25^{+}$Tregs (65).

Indirect strategies of immune suppression. The mechanisms for Treg expansion and conversion are not completely understood but involve cell-to-cell contact (including CD40 and CD4OL interactions) and the production of soluble factors such as TGF- $\beta$, IFN- $\gamma$, and IL-10 (66-68). To sustain the immune-suppressive environment, TAMs and MDSCs secrete an array of chemokines acting on CCR5 and CCR6, which are involved in Treg recruitment (67-69). MDSCs also skew macrophages toward an M2 phenotype, characterized by impaired production of functional IL-12, through a cell contact-dependent mechanism (70). The downregulation of IL-12 is further exacerbated by the macrophages themselves, because TAMs stimulate an additional IL-10 release by MDSCs, thereby creating a self-perpetuating negative loop. Therefore, both MDSCs and TAMs can regulate the intratumoral IL-10/IL-12 balance, which is critical for priming T lymphocyte responses, as reviewed elsewhere $(54,71-73)$. Interestingly, IL-10 receptor blockade enhanced tumor responses to paclitaxel and carboplatin, enabling $\mathrm{CD} 103^{+} \mathrm{DCs}$ to produce IL-12 and support antitumor $\mathrm{CD}^{+} \mathrm{T}$ cells (74).

Direct immune suppression strategies. Direct immune-suppressive mechanisms rely on the activity of enzymes, chemokines, and receptors in myeloid cells. L-arginine and L-tryptophan consumption - which is dependent on the activity of ARG1 (73) and iNOS (75) or indoleamine 2,3-dioxygenase 1 (IDO1) and IDO2 (76), respectively - or L-cysteine deprivation (77) promotes T cell proliferation arrest and functional inhibition by downregulation of the CD3 $\zeta$ chain in the $\mathrm{T}$ cell receptor (TCR) complex. The production of $\mathrm{NO}$ can inhibit $\mathrm{T}$ cell signaling downstream of IL-2R and induce $\mathrm{T}$ cell apoptosis by different mechanisms in an antigen-independent manner $(78,79)$. Another TAM/MDSC-related immune-suppressive mechanism is based on the production of ROS and reactive nitrogen species (RNS). ROS comprise superoxide anion $\left(\mathrm{O}_{2}^{-}\right)$and hydrogen peroxide $\left(\mathrm{H}_{2} \mathrm{O}_{2}\right)$ and are generated in high amounts by the activity of NADPH oxidase (NOX) family members, in which NOX2 is the key player (80). ROS affect T cell fitness by downregulating $\mathrm{CD} 3 \zeta$ chain expression and reducing cytokine secretion, as observed in pancreatic cancer (81). RNS, such as peroxynitrite $\left(\mathrm{ONOO}^{-}\right)$, are byproducts of the combined activity of iNOS, ARG1, and NOX2 and can alter the formation of a correct peptide-MHC complex in MHCI molecules or induce modification of the immunodominant tumor-antigen peptides, thereby affecting TCR recognition and T cell activation (82). RNS can act on $\alpha$ and $\beta$ chains of the TCR, promoting dissociation of the CD3 $\zeta$ chain from the TCR complex and preventing TCR signaling (83). Last, RNS also modify trafficking of leukocytes that promote homing of immune-suppressive subsets (but not $\mathrm{T}$ cells) through aromatic amino acid nitration and nitrosylation of chemokines (CCL2, CCL5, CCL21, CXCL12) or chemokine receptors (CXCR4) $(84,85)$. Myeloid cells also promote immune dysfunction by expressing membrane surface ligands of $\mathrm{T}$ cell-inhibitory receptors, such as programmed death ligand $1 / 2$ (PD-L1/2), which bind programmed death 1 (PD-1) (86-88) and B7-1/2, which bind to cytotoxic T lymphocyte antigen 4 (CTLA4) (89) and CD28 as well as FASL (90). Moreover, TAMs express nonclassical HLA-G and HLA-E molecules that can inhibit T cell activation upon their ligation to the inhibitory leukocyte Ig-like receptor LIT-2 (91).

\section{MDSC- and TAM-dependent protumoral aid}

Cancer stemness. MDSCs finely tune tumor senescence by promoting cellular stemness. At tumor onset in different autochthonous tumor models, neoplastic cells showed a senescent phenotype, a condition limiting tumor progression that was reversed by MDSCs (92). MDSC-secreted IL-1RA was the main molecular mediator of this reprogramming activity, and interference with MDSC trafficking to the tumor (i.e., by CXCL1/2 and CXCR2 targeting) enhanced chemotherapy-induced cellular senescence (92). In human ovarian carcinoma, MDSCs regulated senescence by inducing tumor cell expression of miR-101, which downregulated the stemness repressor C-terminal-binding protein 2 (CTBP2), ultimately triggering cancer stem cell (CSC) sphere formation and enhancing metastatic potential (93). Finally, in a mouse model of pancreatic cancer, MO-MDSCs directly induced expansion of aldehyde dehydrogenase $1^{+}\left(\mathrm{ALDH} 1 \mathrm{~A} 1^{+}\right)$pancreatic CSCs; a similar effect was observed with human CD14 ${ }^{+} \mathrm{HLA}_{-} \mathrm{DR}^{-}$MDSCs from patients with PDAC (94).

In pancreatic tumors, TAM depletion arrests the proliferation of tumor-initiating cells (95). Indeed, TAMs can sustain CSC proliferation by releasing proinflammatory cytokines such as TNF- $\alpha$ and IL-6, which reinforce tumor cell proliferation through NF- $\mathrm{B}$ and STAT3 signaling pathways $(96,97)$. These same molecular pathways may be activated through a direct TAM-to-CSC contact via CD90 and ephrin A4 receptors (98). Finally, the crosstalk between CSCs and TAMs induced TAM secretion of milk fat globule EGF factor 8 (MFGE8) and IL-6, which favored CSC reservoir survival during chemotherapeutic treatment (99).

Angiogenesis. MDSCs and TAMs play a crucial role in promoting the angiogenic switch. During hypoxia adaptation, tumor cells, which sense $\mathrm{O}_{2}$ levels through HIF prolyl hydroxylase 1-3 (PHD1-3) to control HIF-1 $\alpha$ stability, release VEGF and thereby stimulate angiogenesis (100). Similarly, TAMs, in response to hypoxia, release mediators such as VEGF, bFGF, CXCL8/IL-8, and glycolytic enzymes $(101,102)$. Secreted VEGF also orchestrates peripheral expansion, trafficking, and functional commitment of MDSCs (103). In the tumor microenvironment, TAMs and MDSCs release proteases (cathepsin and MMP9), which support angiogenesis by freeing heparin-bound growth factors, such as VEGF-A, and by inducing extracellular matrix remodeling, which promotes invasion (51). Recruitment of MDSCs mediates resistance to anti-VEGF Ab-mediated therapy, as MDSCs can support new vessel growth, even in the presence of anti-VEGF Ab (104), by releasing the proangiogenic bombina variegata peptide 8 (105).

EMT-mesenchymal-to-epithelial transition and metastatic spreading. Myeloid cells play an active role in promoting the spread of distal tumor cells. In mammary tumors, TAMs promote metastatic diffusion via a paracrine loop involving CSF1 and EGF, which induces macrophages and tumor cells to cluster around blood vessels, where macrophages create a gate for tumor cell intravasation into the circulation, thus producing a tumor microenvironment for metastasis (TMEM) (106-108). The proinflammatory proteins S100A8 and S100A9, potent MDSC 
Table 1. Synopsis of therapeutic interventions to limit monocyte and macrophage protumoral activity

\begin{tabular}{|c|c|c|c|}
\hline Drug & Type of cancer & Effects on myeloid cells & References \\
\hline 5-Fluorouracyl & Thymoma (mouse) & MO-MDSC apoptosis & 29,141 \\
\hline Aptamers targeting CD124 (IL-4R $\alpha)$ & Mammary cancer (mouse) & MO-MDSC and TAM depletion & 58 \\
\hline CSF1R antagonist & $\begin{array}{l}\text { Prostate tumor lung carcinoma, diffuse-type giant } \\
\text { cell tumor, and tenosynovial giant cell tumors, } \\
\text { glioma (mouse and human) }\end{array}$ & MO-MDSC expansion and TAM recruitment & $140,152,156$ \\
\hline $\begin{array}{l}\text { Lipid nanoparticles delivering } \\
\text { CCR2-targeting siRNA }\end{array}$ & Thymoma and CRC (mouse) & Ly6Chi inflammatory monocytes and TAM depletion & 159 \\
\hline Bisphosphonates & Mammary tumor (mouse) & TAM depletion, inhibition of MDSC expansion & 160,161 \\
\hline $\begin{array}{l}\text { Combined therapy with IL-12, IL-16, } \\
\text { CpG DNA, and anti-IL-10R mAb }\end{array}$ & Lung and breast cancer (mouse) & TAM reprogramming ${ }^{A}$ & 150 \\
\hline Histidine-rich glycoprotein & Pancreatic and breast cancer, fibrosarcoma (mouse) & TAM reprogramming ${ }^{A}$ & 158 \\
\hline Trabectedin & $\begin{array}{l}\text { Lung and ovarian carcinomas, soft tissue sarcoma } \\
\text { (mouse and human) }\end{array}$ & MO-MDSCs and macrophage depletion & 90 \\
\hline
\end{tabular}

chemoattractants, have been implicated in tumor progression (109); S100A8/A9-induced serum amyloid A3 directly recruited MDSCs to premetastatic lungs, stimulated NF- $\kappa \mathrm{B}$ signaling in a TLR4-dependent manner, and facilitated metastatic spreading (110). Moreover, MO-MDSCs and inflammatory monocytes are recruited through the CCL2/CCR2 axis to a metastatic environment in which they can differentiate into metastasis-associated macrophages (MAMs) $(52,111)$. Hypoxia in primary tumors can trigger MDSC-induced dysfunction in NK cells within the lung premetastatic niche, a defined site to which hematopoietic cells migrate before the tumor cells can seed the niche (112). PMN-MDSCs can also be armed by IL-17 released from $\gamma \delta$ T cells infiltrating the primary breast cancers and assist lymph node and lung metastasis, in part through the inhibition of $\mathrm{CD}^{+} \mathrm{T}$ cell function (113). MDSCs and TAMs also assist the metastatic process by inducing tumor cell EMT. MDSCs attracted by CXCL5 induced EMT in melanoma cells by releasing HGF and TGF- $\beta$ at the primary tumor site; targeting of PMN-MDSCs in this model resulted in marked impairment of primary tumor growth (114). TAM recruitment induces EMT by both TGF- $\beta$ release in a variety of solid tumors (115) and IL-8 in hepatocellular carcinoma (116). Additionally, a positive correlation was found between intratumoral macrophage densities, EMT markers, intraepithelial TGF- $\beta$ levels, and tumor grade of non-small-cell lung cancer (NSCLC) patient samples (115). Because metastatic cells reacquire morphological and phenotypic traits of epithelial cells at the metastatic site, it is conceivable that premetastatic myeloid cells also control a mesenchymal-to-epithelial transition (MET) that promotes cancer cell colonization of and survival in the new organ, likely by releasing the proteoglycan versican (117).

\section{Prognostic significance of myeloid cells in cancer patients}

Three main myeloid classes with distinct lineage commitments have been identified in the blood of cancer patients: monocytic, granulocytic, and immature MDSCs. Each class contains more than one subset (118). Although the role of MDSCs has been acknowledged in primary tumor formation (119), extensive data connect MDSC expansion to more advanced cancer stages (120). MDSC numbers are associated with clinical stage in bladder carcinoma (121), pancreatic adenocarcinoma (122), hepatocellular carcinoma $(123,124)$, gastric cancer (125), NSCLC (126), and head and neck squamous cell carcinoma (127), as well as in hematological malignancies such as non-Hodgkin lymphoma (128). Collectively, these results indicate that expansion of MDSCs in cancer patients is a general phenomenon accompanying tumor progression. MDSC levels also correlated with response to therapy (126, $129,130)$ or surgery (121); however, a deep analysis of clinical outcome in patients showed that MDSC frequency in blood is associated with prognosis, independent of tumor burden $(131,132)$. In patients with either stage IV breast cancer or stage IV colorectal cancer (CRC), a significant correlation was observed between high numbers of circulating MDSCs and poor prognosis. In fact, survival estimates for patients with high numbers of immature MDSCs (lineage ${ }^{-} \mathrm{HLA}-\mathrm{DR}{ }^{\mathrm{lo} /-} \mathrm{CD} 11 \mathrm{~b}^{+} \mathrm{CD} 33^{+}$) in the blood prior to starting standard chemotherapy were associated with shorter overall survival (OS) (133). Finally, high levels of MDSCs, cytokines, and chemokines (PDGF, IL-4, IL-8, IL-17, FGF-2, CCL5, and VEGF) in patients with PDAC are associated with progressive disease (134).

In recent years, immunotherapy has emerged as a therapeutic option for the treatment of cancer. IMA901 is a therapeutic vac- 
cine for RCC that consists of HLA-A ${ }^{\star}$ O2-restricted, tumor-derived peptides. In patients with advanced RCC, the levels of five of six MDSC subsets were expanded at baseline, and two of these subsets were prognostic for OS following IMA901 administration. These results indicate that MDSCs are potential biomarkers of response to the vaccine (135).

Immune checkpoint inhibitors represent a new drug category that is dramatically changing the treatment options for cancer (136). Lower MDSC frequencies correlated with prolonged OS in ipilimumab-treated patients $(132,137)$, whereas a decrease in MDSCs after treatment correlated with improved progressionfree survival (PFS) in advanced melanoma patients receiving neoadjuvant ipilimumab (138). To date, it is not clear whether ipilimumab targets MDSCs or, conversely, whether the lower MDSC levels observed following ipilimumab treatment simply reflect tumor shrinkage in response to immune-mediated rejection.

While some studies demonstrated a correlation of extensive TAM infiltration with poor prognosis in breast, cervix, and bladder carcinomas, conflicting results were obtained in other solid tumors like prostate, NSCLC, and brain cancers (139). Along the same line, a recent meta-analysis of the literature showed inconsistent results (6), since elevated TAM numbers were associated with worse OS in patients with gastric, urogenital, or head and neck cancers, but with better prognosis in patients with CRC.

It appears that, while the expansion of MDSCs is often associated with poor prognosis, expansion of TAMs is not always a negative prognostic factor. When TAM evaluation is carried out at the molecular level, another layer of complexity appears. As discussed above, monocytes from patients with RCC have a distinct transcriptional profile, with upregulation of protumor and antitumor genes. The tumor-promoting function of RCC monocytes and TAMs required IL-1/IL-1R signaling, which also supported progression of RCC xenografts (41). These results are the first indication in human cancers that TAM induction is not mediated by the tumor microenvironment and suggest that patients' monocytes are already primed in the blood. Finally, CSF1R inhibition in a mouse model of proneural glioblastoma (GBM) increased survival by inducing regression of established tumors. Interestingly, a gene signature induced by CSF1R inhibition in murine TAMs was associated with increased survival in patients with proneural GBM (140).

\section{Conclusions and future perspective}

Targeting MO-MDSCs and TAMs can open new therapeutic opportunities to control tumor progression and block metastatic diffusion. The main strategies used thus far involve the inhibition of recruitment, depletion, or reprogramming of target cell populations. Some first-generation chemotherapeutic agents, such as 5 -fluorouracil (141) and gemcitabine $(29,142)$, are able to control MO-MDSC accumulation, probably because these cells are more sensitive than tumor cells to low-dose chemotherapy (29). Lowdose irradiation also increases $\mathrm{CD} 8^{+} \mathrm{T}$ cell trafficking and normalizes tumor vasculature in many cancer models by reprogramming
TAMs toward a more inflammatory M1 type that releases NO (143). However, TAMs can either positively or negatively influence the antitumor activity of cytotoxic chemotherapy and radiotherapy (144), and targeting of immunosuppressive myeloid cells can have different effects on cancer progression $(145,146)$. Additionally, the microbiome can condition different myeloid cells, including TAMs, within murine tumors to contribute to the antitumor efficacy of both chemotherapy and immunotherapy $(147,148)$. Novel biologic drugs recognizing MDSC and TAM antigens or disrupting their function have been developed for selective targeting of these cell populations. As shown in Table 1, these compounds include Abs and/or aptamers $(58,111,149-151)$ as well as molecular antagonists of essential receptors and/or molecular pathways (152). Among chemokines, targeting of CCL2 with a mAb (carlumab, CNTO 888 ) has proven to be beneficial in patients $(153,154)$; however, abrupt discontinuation of the therapy may result in a rebound effect causing increased metastatic disease (155). The inhibition of the CSF1/CSF1R axis with Abs (RG7155) or RTK inhibitors (imatinib mesylate) affects macrophage recruitment and differentiation and has shown encouraging results in clinical trials $(149,156)$. Considering the role of macrophages in regulating the tissue architecture and in mediating innate immune defense, there are concerns about side effects from the extended depletion of these cells. In this context, Abs activating immune stimulators (CD40), combinations of cytokines and Abs, or administration of histidine-rich glycoprotein appeared to modify macrophage polarization toward an antitumor phenotype, without affecting overall macrophage levels $(150,151,157,158)$.

Future investigations will need to focus on the mechanisms driving macrophage polarization toward either proimmune or protumoral phenotypes. Gene expression, proteomic, and metabolomic profiles are increasing our understanding of TAM and MDSC biology and offer potential therapeutic strategies for impeding tumor-induced immune dysfunctions. The identification of functional markers could guide the development of a new class of drugs targeting specific subsets of macrophages and MDSCs, thereby reducing the side effects of ablative therapy. In conclusion, while MDSC/TAM targeting will likely be insufficient to eradicate tumors, interference with patients' immune dysfunctions is a prerequisite and fundamental step for improving the efficacy of passive and active immunotherapeutic protocols.

\section{Acknowledgments}

This work was supported by grants from the Italian Ministry of Health; the Italian Ministry of Education, Universities and Research (FIRB cup: B31J11000420001); the Italian Association for Cancer Research (AIRC) $(6599,12182,14103$, and 12886); and the Fondazione Cassa di Risparmio di Verona, Vicenza, Belluno e Ancona.

Address correspondence to: Vincenzo Bronte, University Hospital and Department of Pathology, Immunology Section, Verona, Italy. Phone: 39.045.8124007; E-mail: vincenzo.bronte@univr.it.
1. Mantovani A, Allavena P, Sica A, Balkwill

F. Cancer-related inflammation. Nature.

2008;454(7203):436-444.

2. Mangerich A, Dedon PC, Fox JG, Tannenbaum
SR, Wogan GN. Chemistry meets biology in

colitis-associated carcinogenesis. Free Radic Res. 2013;47(11):958-986.

3. Schwabe RF, Jobin C. The microbiome and can- cer. Nat Rev Cancer. 2013;13(11):800-812.

4. Balkwill FR, Mantovani A. Cancer-related inflammation: common themes and therapeutic opportunities. Semin Cancer Biol. 2012;22(1):33-40. 
5. Noy R, Pollard JW. Tumor-associated macrophages: from mechanisms to therapy. Immunity. 2014;41(1):49-61.

6. Zhang QW, et al. Prognostic significance of tumor-associated macrophages in solid tumor: a meta-analysis of the literature. PLoS One. 2012;7(12):e50946.

7. Montero AJ, Diaz-Montero CM, Kyriakopoulos CE, Bronte V, Mandruzzato S. Myeloid-derived suppressor cells in cancer patients: a clinical perspective. J Immunother. 2012;35(2):107-115.

8. Yu J, et al. Myeloid-derived suppressor cells suppress antitumor immune responses through IDO expression and correlate with lymph node metastasis in patients with breast cancer. J Immunol. 2013;190(7):3783-3797.

9. Geissmann F, Jung S, Littman DR. Blood monocytes consist of two principal subsets with distinct migratory properties. Immunity. 2003;19(1):71-82.

10. Lesokhin AM, et al. Monocytic CCR2(+) myeloid-derived suppressor cells promote immune escape by limiting activated CD8 T-cell infiltration into the tumor microenvironment. Cancer Res. 2012;72(4):876-886.

11. Schreiber RD, Old LJ, Smyth MJ. Cancer immunoediting: integrating immunity's roles in cancer suppression and promotion. Science. 2011;331(6024):1565-1570.

12. Manz MG, Boettcher S. Emergency granulopoiesis. Nat Rev Immunol. 2014;14(5):302-314.

13. Gomez Perdiguero E, et al. Tissue-resident macrophages originate from yolk-sacderived erythro-myeloid progenitors. Nature. 2015;518(7540):547-551.

14. Hashimoto D, et al. Tissue-resident macrophages self-maintain locally throughout adult life with minimal contribution from circulating monocytes. Immunity. 2013;38(4):792-804.

15. Hoeffel G, et al. C-myb(+) erythro-myeloid progenitor-derived fetal monocytes give rise to adult tissue-resident macrophages. Immunity. 2015;42(4):665-678.

16. Okabe Y, Medzhitov R. Tissue-specific signals control reversible program of localization and functional polarization of macrophages. Cell. 2014;157(4):832-844.

17. Kohyama M, et al. Role for Spi-C in the development of red pulp macrophages and splenic iron homeostasis. Nature. 2009;457(7227):318-321.

18. Murdoch C, Muthana M, Coffelt SB, Lewis $\mathrm{CE}$. The role of myeloid cells in the promotion of tumour angiogenesis. Nat Rev Cancer. 2008;8(8):618-631.

19. Gosselin D, et al. Environment drives selection and function of enhancers controlling tissue-specific macrophage identities. Cell. 2014;159(6):1327-1340.

20. Lavin Y, et al. Tissue-resident macrophage enhancer landscapes are shaped by the local microenvironment. Cell. 2014;159(6):1312-1326.

21. Bain CC, et al. Constant replenishment from circulating monocytes maintains the macrophage pool in the intestine of adult mice. Nat Immunol. 2014;15(10):929-937.

22. Franklin RA, et al. The cellular and molecular origin of tumor-associated macrophages. Science. 2014;344(6186):921-925.
23. Galon J, et al. Type, density, and location of immune cells within human colorectal tumors predict clinical outcome. Science. 2006;313(5795):1960-1964.

24. Cortez-Retamozo V, et al. Origins of tumor-associated macrophages and neutrophils. Proc Natl Acad Sci U S A. 2012;109(7):2491-2496.

25. Shand FH, et al. Tracking of intertissue migration reveals the origins of tumor-infiltrating monocytes. Proc Natl Acad Sci U S A. 2014;111(21):7771-7776.

26. Rossner S, Voigtlander C, Wiethe C, Hanig J, Seifarth C, Lutz MB. Myeloid dendritic cell precursors generated from bone marrow suppress $\mathrm{T}$ cell responses via cell contact and nitric oxide production in vitro. Eur J Immunol. 2005;35(12):3533-3544.

27. Kusmartsev S, Gabrilovich DI. Effect of tumor-derived cytokines and growth factors on differentiation and immune suppressive features of myeloid cells in cancer. Cancer Metastasis Rev. 2006;25(3):323-331.

28. Marigo I, et al. Tumor-induced tolerance and immune suppression depend on the C/EBP $\beta$ transcription factor. Immunity. 2010;32(6):790-802 .

29. Ugel S, et al. Immune tolerance to tumor antigens occurs in a specialized environment of the spleen. Cell Rep. 2012;2(3):628-639.

30. Marvel D, Gabrilovich DI. Myeloid-derived suppressor cells in the tumor microenvironment: expect the unexpected.J Clin Invest. 2015;125(9):3356-3364.

31. Dolcetti L, et al. Hierarchy of immunosuppressive strength among myeloid-derived suppressor cell subsets is determined by GM-CSF. Eur J Immunol. 2010;40(1):22-35.

32. Movahedi K, et al. Identification of discrete tumor-induced myeloid-derived suppressor cell subpopulations with distinct $\mathrm{T}$ cell-suppressive activity. Blood. 2008;111(8):4233-4244.

33. Youn JI, Nagaraj S, Collazo M, Gabrilovich DI. Subsets of myeloid-derived suppressor cells in tumor-bearing mice. J Immunol. 2008;181(8):5791-5802.

34. Wynn TA, Chawla A, Pollard JW. Macrophage biology in development, homeostasis and disease. Nature. 2013;496(7446):445-455.

35. Corzo CA, et al. HIF-1 $\alpha$ regulates function and differentiation of myeloid-derived suppressor cells in the tumor microenvironment. JExp Med. 2010;207(11):2439-2453.

36. Colegio OR, et al. Functional polarization of tumour-associated macrophages by tumourderived lactic acid. Nature. 2014;513(7519):559-563.

37. Hammami I, Chen J, Murschel F, Bronte V, De Crescenzo G, Jolicoeur M. Immunosuppressive activity enhances central carbon metabolism and bioenergetics in myeloid-derived suppressor cells in vitro models. BMC Cell Biol. 2012;13:18.

38. Movahedi K, et al. Different tumor microenvironments contain functionally distinct subsets of macrophages derived from Ly6C (high) monocytes. Cancer Res. 2010;70(14):5728-5739.

39. Murray PJ, et al. Macrophage activation and polarization: nomenclature and experimental guidelines. Immunity. 2014;41(1):14-20.

40. Cavnar MJ, et al. KIT oncogene inhibition drives intratumoral macrophage M2 polarization. JExp Med. 2013;210(13):2873-2886.

41. Chittezhath M, et al. Molecular profiling reveals a tumor-promoting phenotype of monocytes and macrophages in human cancer progression. Immunity. 2014;41(5):815-829.

42. Casbon AJ, et al. Invasive breast cancer reprograms early myeloid differentiation in the bone marrow to generate immunosuppressive neutrophils. Proc Natl Acad Sci U S A. 2015;112(6):E566-E575.

43. Kowanetz M, et al. Granulocyte-colony stimulating factor promotes lung metastasis through mobilization of Ly $6 \mathrm{G}^{+} \mathrm{Ly} 6 \mathrm{C}^{+}$granulocytes. Proc Natl Acad Sci U S A. 2010;107(50):21248-21255.

44. Clark CE, Hingorani SR, Mick R, Combs C, Tuveson DA, Vonderheide RH. Dynamics of the immune reaction to pancreatic cancer from inception to invasion. Cancer Res. 2007;67(19):9518-9527.

45. Damuzzo V, et al. Complexity and challenges in defining myeloid-derived suppressor cells. Cytometry B Clin Cytom. 2015;88(2):77-91.

46. Khaled YS, Ammori BJ, Elkord E. Increased levels of granulocytic myeloid-derived suppressor cells in peripheral blood and tumour tissue of pancreatic cancer patients. JImmunol Res. 2014;2014:879897.

47. Bayne LJ, et al. Tumor-derived granulocyte-macrophage colony-stimulating factor regulates myeloid inflammation and $\mathrm{T}$ cell immunity in pancreatic cancer. Cancer Cell. 2012;21(6):822-835.

48. Schmidt K, Zilio S, Schmollinger JC, Bronte V, Blankenstein T, Willimsky G. Differently immunogenic cancers in mice induce immature myeloid cells that suppress CTL in vitro but not in vivo following transfer. Blood. 2013;121(10):1740-1748.

49. Su S, et al. A positive feedback loop between mesenchymal-like cancer cells and macrophages is essential to breast cancer metastasis. Cancer Cell. 2014;25(5):605-620.

50. Lin EY, Nguyen AV, Russell RG, Pollard JW. Colony-stimulating factor 1 promotes progression of mammary tumors to malignancy. J Exp Med. 2001;193(6):727-740.

51. Quail DF, Joyce JA. Microenvironmental regulation of tumor progression and metastasis. Nat Med. 2013;19(11):1423-1437.

52. Qian BZ, Pollard JW. Macrophage diversity enhances tumor progression and metastasis. Cell. 2010;141(1):39-51.

53. Balkwill F. Cancer and the chemokine network. Nat Rev Cancer. 2004;4(7):540-550.

54. Allavena P, Mantovani A. Immunology in the clinic review series; focus on cancer: tumourassociated macrophages: undisputed stars of the inflammatory tumour microenvironment. Clin Exp Immunol.2012;167(2):195-205.

55. Gray MJ, Poljakovic M, Kepka-Lenhart D, Morris SM, Morris SM Jr. Induction of arginase I transcription by IL-4 requires a composite DNA response element for STAT6 and C/EBP $\beta$. Gene. 2005;353(1):98-106.

56. Kohanbash G, et al. GM-CSF promotes the immunosuppressive activity of glioma-infiltrating myeloid cells through interleukin- 4 receptor- $\alpha$. Cancer Res. 2013;73(21):6413-6423.

57. Gallina G, et al. Tumors induce a subset of 
inflammatory monocytes with immunosuppressive activity on $\mathrm{CD}^{+} \mathrm{T}$ cells. J Clin Invest. 2006;116(10):2777-2790.

58. Roth F, De La Fuente AC, Vella JL, Zoso A, Inverard L, Serafini P. Aptamer-mediated blockade of IL 4 R $\alpha$ triggers apoptosis of MDSCs and limits tumor progression. Cancer Res. 2012;72(6):1373-1383.

59. Gocheva V, et al. IL-4 induces cathepsin protease activity in tumor-associated macrophages to promote cancer growth and invasion. Genes Dev. 2010;24(3):241-255.

60. DeNardo DG, et al. CD4(+) T cells regulate pulmonary metastasis of mammary carcinomas by enhancing protumor properties of macrophages. Cancer Cell. 2009;16(2):91-102.

61. Hanahan D, Coussens LM. Accessories to the crime: functions of cells recruited to the tumor microenvironment. Cancer Cell. 2012;21(3):309-322.

62. Srivastava MK, Bosch JJ, Thompson JA, Ksander BR, Edelman MJ, Ostrand-Rosenberg S. Lung cancer patients' CD4(+) T cells are activated in vitro by MHC II cell-based vaccines despite the presence of myeloid-derived suppressor cells. Cancer Immunol Immunother. 2008;57(10):1493-1504.

63. Mandruzzato S, et al. IL $4 \mathrm{R} \alpha^{+}$myeloid-derived suppressor cell expansion in cancer patients. J Immunol. 2009;182(10):6562-6568.

64. Bennett JA, Rao VS, Mitchell MS. Systemic bacillus Calmette-Guerin (BCG) activates natural suppressor cells. Proc Natl Acad Sci U S A. 1978;75(10):5142-5144.

65. Savage ND, et al. Human anti-inflammatory macrophages induce Foxp $3^{+} \mathrm{GITR}^{+} \mathrm{CD} 25^{+}$regulatory $\mathrm{T}$ cells, which suppress via membrane-bound TGFß-1. JImmunol. 2008;181(3):2220-2226.

66. Serafini P, Mgebroff S, Noonan K, Borrello I. Myeloid-derived suppressor cells promote cross-tolerance in B-cell lymphoma by expanding regulatory T cells. Cancer Res. 2008;68(13):5439-5449.

67. Curiel TJ, et al. Specific recruitment of regulatory $\mathrm{T}$ cells in ovarian carcinoma fosters immune privilege and predicts reduced survival. Nat Med. 2004;10(9):942-949.

68. Liu J, et al. Tumor-associated macrophages recruit $\mathrm{CCR}^{+}{ }^{+}$regulatory $\mathrm{T}$ cells and promote the development of colorectal cancer via enhancing CCL2O production in mice. PLoS One. 2011;6(4):e19495.

69. Schlecker E, et al. Tumor-infiltrating monocytic myeloid-derived suppressor cells mediate CCR5-dependent recruitment of regulatory $\mathrm{T}$ cells favoring tumor growth. J Immunol. 2012;189(12):5602-5611.

70. Sinha P, Clements VK, Bunt SK, Albelda SM, Ostrand-Rosenberg S. Cross-talk between myeloid-derived suppressor cells and macrophages subverts tumor immunity toward a type 2 response. J Immunol. 2007;179(2):977-983.

71. Sica A, Bronte V. Altered macrophage differentiation and immune dysfunction in tumor development. J Clin Invest. 2007;117(5):1155-1166.

72. Ostrand-Rosenberg S, Sinha P, Beury DW, Clements VK. Cross-talk between myeloid-derived suppressor cells (MDSC), macrophages, and dendritic cells enhances tumor-induced immune suppression. Semin Cancer Biol. 2012;22(4):275-281.

73. Biswas SK, Mantovani A. Macrophage plasticity and interaction with lymphocyte subsets: cancer as a paradigm. Nat Immunol. 2010;11(10):889-896.

74. Ruffell B, et al. Macrophage IL-10 blocks CD8 ${ }^{+}$ $\mathrm{T}$ cell-dependent responses to chemotherapy by suppressing IL-12 expression in intratumoral dendritic cells. Cancer Cell. 2014;26(5):623-637.

75. Bronte V, Zanovello P. Regulation of immune responses by L-arginine metabolism. Nat Rev Immunol. 2005;5(8):641-654.

76. Munn DH, et al. GCN2 kinase in T cells mediates proliferative arrest and anergy induction in response to indoleamine 2,3-dioxygenase. Immunity. 2005;22(5):633-642

77. Srivastava MK, Sinha P, Clements VK, Rodriguez P, Ostrand-Rosenberg S. Myeloid-derived suppressor cells inhibit T-cell activation by depleting cystine and cysteine. Cancer Res. 2010;70(1):68-77.

78. Mazzoni A, et al. Myeloid suppressor lines inhibit $\mathrm{T}$ cell responses by an NO-dependent mechanism. JImmunol. 2002;168(2):689-695.

79. Macphail SE, Gibney CA, Brooks BM, Booth CG, Flanagan BF, Coleman JW. Nitric oxide regulation of human peripheral blood mononuclear cells: critical time dependence and selectivity for cytokine versus chemokine expression. JImmunol. 2003;171(9):4809-4815.

80. Corzo CA, et al. Mechanism regulating reactive oxygen species in tumor-induced myeloid-derived suppressor cells. J Immunol. 2009;182(9):5693-5701.

81. Schmielau J, Nalesnik MA, Finn OJ. Suppressed T-cell receptor zeta chain expression and cytokine production in pancreatic cancer patients. Clin Cancer Res. 2001;7(3):933s-939s.

82. Hardy LL, Wick DA, Webb JR. Conversion of tyrosine to the inflammation-associated analog 3'-nitrotyrosine at either TCR- or MHC-contact positions can profoundly affect recognition of the MHC class I-restricted epitope of lymphocytic choriomeningitis virus glycoprotein 33 by CD $8 \mathrm{~T}$ cells. J Immunol. 2008;180(9):5956-5962.

83. Nagaraj S, Schrum AG, Cho HI, Celis E, Gabrilovich DI. Mechanism of T cell tolerance induced by myeloid-derived suppressor cells. J Immunol. 2010;184(6):3106-3116

84. De Sanctis F, et al. The emerging immunological role of post-translational modifications by reactive nitrogen species in cancer microenvironment. Front Immunol. 2014;5:69.

85. Molon B, et al. Chemokine nitration prevents intratumoral infiltration of antigen-specific $\mathrm{T}$ cells. JExp Med. 2011;208(10):1949-1962.

86. Duraiswamy J, Freeman GJ, Coukos G. Therapeutic PD-1 pathway blockade augments with other modalities of immunotherapy T-cell function to prevent immune decline in ovarian cancer. Cancer Res. 2013;73(23):6900-6912.

87. Loke P, Allison JP. PD-L1 and PD-L2 are differentially regulated by Th1 and Th2 cells. Proc Natl Acad Sci U S A. 2003;100(9):5336-5341.

88. Noman MZ, et al. PD-L1 is a novel direct target of HIF-1 $\alpha$, and its blockade under hypoxia enhanced MDSC-mediated T cell activation. J Exp Med. 2014;211(5):781-790.

89. Kennedy BC, et al. Tumor-associated macrophages in glioma: friend or foe? J Oncol. 2013;2013:486912.

90. Germano G, et al. Role of macrophage targeting in the antitumor activity of trabectedin. Cancer Cell. 2013;23(2):249-262.

91. LeMaoult J, Krawice-Radanne I, Dausset J, Carosella ED. HLA-G1-expressing antigen-presenting cells induce immunosuppressive CD $4^{+}$T cells. Proc Natl Acad Sci U S A. 2004;101(18):7064-7069.

92. Di Mitri D, et al. Tumour-infiltrating Gr-1 ${ }^{+}$ myeloid cells antagonize senescence in cancer. Nature. 2014;515(7525):134-137.

93. Cui TX, et al. Myeloid-derived suppressor cells enhance stemness of cancer cells by inducing microRNA101 and suppressing the corepressor CtBP2. Immunity. 2013;39(3):611-621.

94. Panni RZ, et al. Tumor-induced STAT3 activation in monocytic myeloid-derived suppressor cells enhances stemness and mesenchymal properties in human pancreatic cancer. Cancer Immunol Immunother. 2014;63(5):513-528.

95. Mitchem JB, et al. Targeting tumor-infiltrating macrophages decreases tumor-initiating cells, relieves immunosuppression, and improves chemotherapeutic responses. Cancer Res. 2013;73(3):1128-1141.

96. Schwitalla S, et al. Intestinal tumorigenesis initiated by dedifferentiation and acquisition of stem-cell-like properties. Cell. 2013;152(1):25-38.

97. Wan S, et al. Tumor-associated macrophages produce interleukin 6 and signal via STAT3 to promote expansion of human hepatocellular carcinoma stem cells. Gastroenterology 2014;147(6):1393-1404.

98. Lu H, et al. A breast cancer stem cell niche supported by juxtacrine signalling from monocytes and macrophages. Nat Cell Biol. 2014;16(11):1105-1117.

99. Jinushi M, et al. Tumor-associated macrophages regulate tumorigenicity and anticancer drug responses of cancer stem/initiating cells. Proc Natl Acad Sci U S A. 2011;108(30):12425-12430.

100.Mukhopadhyay D, Tsiokas L, Zhou XM, Foster D, Brugge JS, Sukhatme VP. Hypoxic induction of human vascular endothelial growth factor expression through c-Src activation. Nature. 1995;375(6532):577-581.

101. Murdoch C, Giannoudis A, Lewis CE. Mechanisms regulating the recruitment of macrophages into hypoxic areas of tumors and other ischemic tissues. Blood. 2004;104(8):2224-2234.

102. Schmidt T, Carmeliet P. Blood-vessel formation: bridges that guide and unite. Nature. 2010;465(7299):697-699.

103. Gabrilovich DI, Ostrand-Rosenberg S, Bronte V. Coordinated regulation of myeloid cells by tumours. Nat Rev Immunol. 2012;12(4):253-268.

104. Shojaei F, et al. Tumor refractoriness to anti-VEGF treatment is mediated by $\mathrm{CD} 11 \mathrm{~b}^{+} \mathrm{Gr} 1^{+}$myeloid cells. Nat Biotechnol. 2007;25(8):911-920.

105. Shojaei F, et al. Bv8 regulates myeloidcell-dependent tumour angiogenesis. Nature. 2007;450(7171):825-831.

106.Condeelis J, Pollard JW. Macrophages: obligate partners for tumor cell migration, invasion, and metastasis. Cell. 2006;124(2):263-266.

107. Wyckoff JB, et al. Direct visualization of macrophage-assisted tumor cell intravasation in mammary tumors. Cancer Res. 2007;67(6):2649-2656. 108. Kitamura T, Qian BZ, Pollard JW. Immune cell 
promotion of metastasis. Nat Rev Immunol. 2015;15(2):73-86.

109. Sinha P, Okoro C, Foell D, Freeze HH, Ostrand-Rosenberg S, Srikrishna G. Proinflammatory $\mathrm{S} 100$ proteins regulate the accumulation of myeloid-derived suppressor cells. J Immunol. 2008;181(7):4666-4675.

110. Hiratsuka S, et al. The S100A8-serum amyloid A3-TLR4 paracrine cascade establishes a pre-metastatic phase. Nat Cell Biol. 2008;10(11):1349-1355.

111. Qian BZ, et al. CCL2 recruits inflammatory monocytes to facilitate breast-tumour metastasis. Nature. 2011;475(7355):222-225.

112. Sceneay J, et al. Primary tumor hypoxia recruits CD11b+/Ly6Cmed/Ly6G+ immune suppressor cells and compromises NK cell cytotoxicity in the premetastatic niche. Cancer Res. 2012;72(16):3906-3911.

113. Coffelt SB, et al. IL-17-producing gammadelta T cells and neutrophils conspire to promote breast cancer metastasis. Nature. 2015;522(7556):345-348.

114. Toh B, et al. Mesenchymal transition and dissemination of cancer cells is driven by myeloidderived suppressor cells infiltrating the primary tumor. PLoS Biol. 2011;9(9):e1001162.

115. Bonde AK, Tischler V, Kumar S, Soltermann A, Schwendener RA. Intratumoral macrophages contribute to epithelial-mesenchymal transition in solid tumors. BMC Cancer. 2012;12:35.

116. Fu XT, et al. Macrophage-secreted IL-8 induces epithelial-mesenchymal transition in hepatocellular carcinoma cells by activating the JAK2/STAT3/Snail pathway. Int JOncol. 2015;46(2):587-596.

117. Gao D, et al. Myeloid progenitor cells in the premetastatic lung promote metastases by inducing mesenchymal to epithelial transition. Cancer Res. 2012;72(6):1384-1394.

118. Solito S, Marigo I, Pinton L, Damuzzo V, Mandruzzato S, Bronte V. Myeloid-derived suppressor cell heterogeneity in human cancers. Ann N Y Acad Sci. 2014;1319:47-65.

119. Talmadge JE, Gabrilovich DI. History of myeloid-derived suppressor cells. Nat Rev Cancer. 2013;13(10):739-752.

120. Diaz-Montero CM, Salem ML, Nishimura MI, Garrett-Mayer E, Cole DJ, Montero AJ. Increased circulating myeloid-derived suppressor cells correlate with clinical cancer stage, metastatic tumor burden, and doxorubicin-cyclophosphamide chemotherapy. Cancer Immunol Immunother. 2009;58(1):49-59.

121. Yuan XK, Zhao XK, Xia YC, Zhu X, Xiao P. Increased circulating immunosuppressive CD14(+)HLA-DR(-/low) cells correlate with clinical cancer stage and pathological grade in patients with bladder carcinoma. J Int Med Res. 2011;39(4):1381-1391.

122. Porembka MR, et al. Pancreatic adenocarcinoma induces bone marrow mobilization of myeloid-derived suppressor cells which promote primary tumor growth. Cancer Immunol Immunother. 2012;61(9):1373-1385.

123. Shen P, Wang A, He M, Wang Q, Zheng S. Increased circulating Lin(-/low) CD33(+) HLA-DR(-) myeloid-derived suppressor cells in hepatocellular carcinoma patients. Hepatol Res. 2014;44(6):639-650.

124. Arihara F, et al. Increase in $\mathrm{CD}^{+} 4^{+} \mathrm{HLA}^{-} \mathrm{DR}^{-} /$ low myeloid-derived suppressor cells in hepatocellular carcinoma patients and its impact on prognosis. Cancer Immunol Immunother. 2013;62(8):1421-1430.

125. Wang L, Chang EW, Wong SC, Ong SM, Chong DQ, Ling KL. Increased myeloid-derived suppressor cells in gastric cancer correlate with cancer stage and plasma S100A8/A9 proinflammatory proteins. JImmunol. 2013;190(2):794-804.

126. Huang A, Zhang B, Wang B, Zhang F, Fan KX Guo YJ. Increased CD14(+)HLA-DR (-/low) myeloid-derived suppressor cells correlate with extrathoracic metastasis and poor response to chemotherapy in non-small cell lung cancer patients. Cancer Immunol Immunother. 2013;62(9):1439-1451.

127. Vasquez-Dunddel D, et al. STAT3 regulates arginase-I in myeloid-derived suppressor cells from cancer patients. J Clin Invest. 2013;123(4):1580-1589.

128. Lin Y, Gustafson MP, Bulur PA, Gastineau DA, Witzig TE, Dietz AB. Immunosuppressive CD14 ${ }^{+}$HLA-DR ${ }^{\text {low/- }}$ monocytes in B-cell non-Hodgkin lymphoma. Blood. 2011; 117(3):872-881.

129. Ko JS, et al. Sunitinib mediates reversal of myeloid-derived suppressor cell accumulation in renal cell carcinoma patients. Clin Cancer Res. 2009;15(6):2148-2157.

130. Liu CY, et al. Population alterations of L-arginaseand inducible nitric oxide synthase-expressed

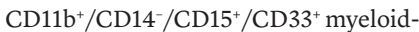
derived suppressor cells and $\mathrm{CD}^{+} \mathrm{T}$ lymphocytes in patients with advanced-stage non-small cell lung cancer. J Cancer Res Clin Oncol. 2010;136(1):35-45.

131. Gabitass RF, Annels NE, Stocken DD, Pandha HA, Middleton GW. Elevated myeloid-derived suppressor cells in pancreatic, esophageal and gastric cancer are an independent prognostic factor and are associated with significant elevation of the Th2 cytokine interleukin-13. Cancer Immunol Immunother. 2011;60(10):1419-1430.

132. Kitano S, et al. Computational algorithm-driven evaluation of monocytic myeloid-derived suppressor cell frequency for prediction of clinical outcomes. Cancer Immunol Res. 2014;2(8):812-821.

133. Solito S, et al. A human promyelocytic-like population is responsible for the immune suppression mediated by myeloid-derived suppressor cells. Blood. 2011;118(8):2254-2265.

134. Markowitz J, et al. Patients with pancreatic adenocarcinoma exhibit elevated levels of myeloid-derived suppressor cells upon progression of disease. Cancer Immunol Immunother. 2015;64(2):149-159.

135. Walter S, et al. Multipeptide immune response to cancer vaccine IMA901 after single-dose cyclophosphamide associates with longer patient survival. Nat Med. 2012;18(8):1254-1261.

136. Page DB, Postow MA, Callahan MK, Allison JP, Wolchok JD. Immune modulation in cancer with antibodies. Annu Rev Med. 2014;65:185-202.

137. Meyer C, et al. Frequencies of circulating MDSC correlate with clinical outcome of melanoma patients treated with ipilimumab. Cancer Immunol Immunother. 2014;63(3):247-257.

138. Tarhini AA, et al. Immune monitoring of the circulation and the tumor microenvironment in patients with regionally advanced melanoma receiving neoadjuvant ipilimumab. PLoS One. 2014;9(2):e87705.

139. Bingle L, Brown NJ, Lewis CE. The role of tumour-associated macrophages in tumour progression: implications for new anticancer therapies. J Pathol. 2002;196(3):254-265.

140. Pyonteck SM, et al. CSF-1R inhibition alters macrophage polarization and blocks glioma progression. Nat Med. 2013;19(10):1264-1272.

141. Vincent J, et al. 5-Fluorouracil selectively kills tumor-associated myeloid-derived suppressor cells resulting in enhanced T celldependent antitumor immunity. Cancer Res. 2010;70(8):3052-3061.

142.Suzuki E, Kapoor V, Jassar AS, Kaiser LR, Albelda SM. Gemcitabine selectively eliminates splenic $\mathrm{Gr}-1^{+} / \mathrm{CD} 11 \mathrm{~b}^{+}$myeloid suppressor cells in tumor-bearing animals and enhances antitumor immune activity. Clin Cancer Res. 2005;11(18):6713-6721.

143. Klug F, et al. Low-dose irradiation programs macrophage differentiation to an iNOS(+)/M1 phenotype that orchestrates effective $\mathrm{T}$ cell immunotherapy. Cancer Cell. 2013;24(5):589-602.

144.Watson GA, Fu YX, Lopez DM. Splenic macrophages from tumor-bearing mice co-expressing MAC- 1 and MAC-2 antigens exert immunoregulatory functions via two distinct mechanisms. JLeukoc Biol. 1991;49(2):126-138.

145. De Palma M, Lewis CE. Macrophage regulation of tumor responses to anticancer therapies. Cancer Cell. 2013;23(3):277-286.

146.Dijkgraaf EM, et al. Chemotherapy alters monocyte differentiation to favor generation of cancersupporting M2 macrophages in the tumor microenvironment. Cancer Res. 2013;73(8):2480-2492.

147. Filipazzi P, et al. Identification of a new subset of myeloid suppressor cells in peripheral blood of melanoma patients with modulation by a granulocyte-macrophage colony-stimulation factor-based antitumor vaccine. J Clin Oncol. 2007;25(18):2546-2553.

148. Diaz-Montero CM, Finke J, Montero AJ. Myeloid-derived suppressor cells in cancer: therapeutic, predictive, and prognostic implications. Semin Oncol. 2014;41(2):174-184.

149. Ries CH, et al. Targeting tumor-associated macrophages with anti-CSF-1R antibody reveals a strategy for cancer therapy. Cancer Cell. 2014;25(6):846-859.

150. Guiducci C, Vicari AP, Sangaletti S, Trinchieri G, Colombo MP. Redirecting in vivo elicited tumor infiltrating macrophages and dendritic cells towards tumor rejection. Cancer Res. 2005;65(8):3437-3446

151. Weiss JM, et al. Macrophage-dependent nitric oxide expression regulates tumor cell detachment and metastasis after IL-2/anti-CD40 immunotherapy. JExp Med. 2010;207(11):2455-2467.

152. Priceman SJ, et al. Targeting distinct tumorinfiltrating myeloid cells by inhibiting CSF-1 receptor: combating tumor evasion of antiangiogenic therapy. Blood. 2010;115(7):1461-1471. 
153. Pienta KJ, et al. Phase 2 study of carlumab (CNTO 888), a human monoclonal antibody against CC-chemokine ligand 2 (CCL2), in metastatic castration-resistant prostate cancer. Invest New Drugs. 2013;31(3):760-768.

154. Sandhu SK, et al. A first-in-human, first-in-class, phase I study of carlumab (CNTO 888), a human monoclonal antibody against CC-chemokine ligand 2 in patients with solid tumors. Cancer Chemother Pharmacol. 2013;71(4):1041-1050.

155. Ortiz ML, et al. Immature myeloid cells directly contribute to skin tumor development by recruiting IL-17-producing CD4 $4^{+} \mathrm{T}$ cells. J Exp Med.
2015;212(3):351-367.

156. Cassier PA, et al. Efficacy of imatinib mesylate for the treatment of locally advanced and/ or metastatic tenosynovial giant cell tumor/ pigmented villonodular synovitis. Cancer. 2012;118(6):1649-1655.

157. Beatty GL, et al. CD40 agonists alter tumor stroma and show efficacy against pancreatic carcinoma in mice and humans. Science. 2011;331(6024):1612-1616.

158. Rolny C, et al. HRG inhibits tumor growth and metastasis by inducing macrophage polarization and vessel normalization through downregula- tion of PlGF. Cancer Cell. 2011;19(1):31-44.

159. Condamine T, Gabrilovich DI. Molecular mechanisms regulating myeloid-derived suppressor cell differentiation and function. Trends Immunol. 2011;32(1):19-25.

160. Brown HK, Holen I. Anti-tumour effects of bisphosphonates--what have we learned from in vivo models? Curr Cancer Drug Targets. 2009;9(7):807-823.

161. Veltman JD, et al. Zoledronic acid impairs myeloid differentiation to tumour-associated macrophages in mesothelioma. Br JCancer. 2010;103(5):629-641. 